\title{
Mindfulness is associated with intrinsic functional connectivity between default mode and salience networks
}

\author{
Anselm Doll ${ }^{1,2,3,4 *}$, Britta K. Hölzel ${ }^{1}$, Christine C. Boucard ${ }^{3,5}$, Afra M. Wohlschläger ${ }^{1,3,4,5}$ \\ and Christian Sorg $1,2,3,6$

\begin{abstract}
'Department of Neuroradiology, Technische Universität München TUM, Munich, Germany, ${ }^{2}$ Department of Psychiatry, Technische Universität München TUM, Munich, Germany, ${ }^{3}$ TUM-Neuro Imaging Center of Klinikum Rechts der Isar, Technische Universität München TUM, Munich, Germany, ${ }^{4}$ Graduate School of Systemic Neurosciences, Ludwig-Maximilians-University, Munich, Germany, ${ }^{5}$ Department of Neurology, Technische Universität München TUM,
\end{abstract} \\ Munich, Germany, ${ }^{6}$ Department of Nuclear Medicine, Technische Universität München TUM, Munich, Germany
}

OPEN ACCESS

Edited by:

Daniel S. Margulies, Max Planck Institute for Human Cognitive and Brain Sciences,

Germany

Reviewed by: Bradford C. Dickerson, Harvard Medical School, USA Lisa Ann Kilpatrick, University of California, Los Angeles,

USA

*Correspondence: Anselm Doll,

Department of Neuroradiology and Psychiatry, TUM-Neuro Imaging Centre of Klinikum Rechts der Isar, Technische Universität München TUM, Ismaninger Str. 22, 81675 München, Germany anselmdol/@gmail.com

Received: 19 January 2015 Accepted: 06 August 2015 Published: 25 August 2015

Citation: Doll A, Hölzel BK, Boucard CC, Wohlschläger AM and Sorg C (2015) Mindfulness is associated with intrinsic functional connectivity between default mode and salience networks.

Front. Hum. Neurosci. 9:461. doi: 10.3389/fnhum.2015.00461
Mindfulness is attention to present moment experience without judgment. Mindfulness practice is associated with brain activity in areas overlapping with the default mode, salience, and central executive networks (DMN, SN, CEN). We hypothesized that intrinsic functional connectivity (iFC; i.e., synchronized ongoing activity) across these networks is associated with mindfulness scores. After 2 weeks of daily 20 min attention to breath training, healthy participants were assessed by mindfulness questionnaires and resting-state functional MRI. Independent component analysis (ICA) of imaging data revealed networks of interest, whose activity time series defined inter-network intrinsic functional connectivity (inter-iFC) by temporal correlation. Inter-iFC between subnetworks of the DMN and SN-and inter-iFC between subnetworks of the SN and left CEN at trend-was correlated with mindfulness scores. Additional control analyses about visual networks' inter-iFC support the specificity of our findings. Results provide evidence that mindfulness is associated with iFC between DMN and SN. Data suggest that ongoing interactions among central intrinsic brain networks link with the ability to attend to current experience without judgment.

Keywords: resting state, connectivity, mindfulness, fMRI, salience network, central executive network, default network

\section{Introduction}

Mindfulness refers to attending to present moment experience without judging occurring feelings or thoughts (Bishop et al., 2004). Mindfulness practice such as meditation has proved beneficial for well-being (Carmody, 2009), and is an efficient element of treatments for mental disorders (Hofmann et al., 2010). Mindfulness practice typically recruits a number of brain regions, mainly prefrontal, parietal and subcortical brain areas (Creswell et al., 2007; Frewen et al., 2010; Hasenkamp et al., 2012; Dickenson et al., 2013). For example, Dickenson et al. (2013) found activations in dorso-medial prefrontal cortex (dmPFC), anterior cingulate cortex (ACC), insula, and temporo-parietal junction during a controlled focused breathing task-the most prominent first technique in teaching mindfulness to novices. Due to its widespread activation pattern, 
distributed functional brain networks have been suggested as critical neural correlates of mindfulness practice. For example, Hasenkamp and Barsalou (2012) recently identified four different mental states during meditation with each state being preferentially related to activity in different intrinsic brain networks: focus on the present experience was most strongly related to dorso-lateral prefrontal cortex activation of the central executive network (CEN), mind wandering was associated with the default mode network (DMN), awareness of mind wandering was linked with activation in the salience network (SN), and a shift of attention back towards focus on the present experience was again linked with the right dorsolateral prefrontal cortex (DLPFC) and right posterior parietal cortex with both regions being part of the CEN.

Intrinsic brain networks such as the DMN, SN, and CEN are characterized by coherent ongoing activity at infra-slow frequencies and are often studied during resting state. These networks are believed to subserve specific cognitive functions like attentional control or core affect (Fox and Raichle, 2007) as their patterns of coherent ongoing activity overlap and reflect the activation patterns observed during goal directed behavior (Lewis et al., 2009; Smith et al., 2009; Berkes et al., 2011; Riedl et al., 2011). Multiple experiments have shown that correlated resting-state activity (i.e., intrinsic functional connectivity-iFC) within the DMN, SN, CEN, respectively, is associated with mindfulness (Kilpatrick et al., 2011; Hasenkamp and Barsalou, 2012; Shaurya Prakash et al., 2013; Taylor et al., 2013; Berkovich-Ohana et al., 2014). However, previous studies focused mainly on within-network functional connectivity, ignoring that additional networks are involved in mindfulness and particularly that these networks interact with each other (see Kilpatrick et al., 2011; Froeliger et al., 2012). Based on Hasenkamp and Barsalou's (2012) model, we hypothesized that the ability of mindfulness is coded in ongoing inter-network interactions between DMN, SN, and CEN. To test this hypothesis we investigated the inter-network functional connectivity during resting state (inter-iFC) of the $\mathrm{SN}, \mathrm{DMN}$, and CEN in 26 healthy controls that were trained in breath awareness for 2 weeks prior to scanning, and correlated these values with participants' mindfulness scores of psychometric assessment.

\section{Materials and Methods}

\section{Participants}

Twenty six right-handed meditation naïve volunteers that were free from past and present neurological and psychiatric disorders participated in the study (10 males, mean age $( \pm S D)=26.9 \pm 4.6)$. The study was approved by the ethical committee of the Technische Universität München. All participants provided written informed consent prior to the experiment and received a monetary compensation of expenses for participation.

\section{Mindfulness Ability}

To ensure mindfulness ability, participants received $20 \mathrm{~min}$ of audio-guided training in attention to breath, based on a meditation as taught in a mindfulness based stress reduction
(MBSR) program (Hölzel, 2012) daily for 2 weeks. Mindfulness ability was measured with the Mindful Attention and Awareness Scale (MAAS; Brown and Ryan, 2003) and the Freiburg Mindfulness Inventory (FMI; Walach et al., 2006). While the MAAS focuses on the presence or absence of awareness of what is occurring in the present, the FMI assesses the accepting and curious attitude towards this experience that is inherent to mindfulness. Both questionnaires have been shown to hold good internal consistency (Cronbach's $\alpha=0.82$ and 0.86 , respectively) and validity.

\section{Functional MRI: Data Acquisition and Analysis}

For imaging, participants were instructed to remain still with eyes closed and to not fall asleep during acquisition. All participants reported to not have fallen asleep during the scanning session.

\section{Imaging Acquisition}

Magnetic resonance imaging was performed on a 3-T whole body MR scanner (Verio, Siemens, Germany) using a standard head coil. For co-registration of functional data, T1-weighted anatomical data were obtained from each subject by using a magnetization-prepared rapid acquisition gradient echo sequence $[\mathrm{MP}-\mathrm{RAGE}$, time to echo $(\mathrm{TE})=4 \mathrm{~ms}$, repetition time $(\mathrm{TR})=9 \mathrm{~ms}$, time for inversion $(\mathrm{TI})=100 \mathrm{~ms}$, flip angle $=5^{\circ}$, field of view $(\mathrm{FoV})=240 \mathrm{~mm} \times 240 \mathrm{~mm}$, matrix $=240 \times 240$, 170 slices, voxel size $=1 \mathrm{~mm} \times 1 \mathrm{~mm} \times 1 \mathrm{~mm}]$. Functional data were collected using a contrast-gradient echo planar imaging $(\mathrm{EPI})$ sequence $\left(\mathrm{TE}=35 \mathrm{~ms}, \mathrm{TR}=2000 \mathrm{~ms}\right.$, flip angle $=90^{\circ}, 35$ slices, slice thickness $=3 \mathrm{~mm}$, and $0 \mathrm{~mm}$ interslice gap).

\section{fMRI Data Analysis}

Preprocessing and analysis of imaging data was carried out with SPM8 (Wellcome Department of Cognitive Neurology, London, UK). After coregistration and segmentation, T1weighted structural images were normalized to a standard T1 template in MNI space with a $1 \times 1 \times 1 \mathrm{~mm}$ resolution. After discarding the first three volumes, preprocessing of T2*-weighted functional images included slice timing, spatial realignment to the first image of the run, normalization to SPM8's EPI template in the Montreal Neurological Institute (MNI) space, resampling to $3 \times 3 \times 3 \mathrm{~mm}$ and smoothing with an $8 \mathrm{~mm}$ full width at half maximum (FWHM) Gaussian filter.

To define intrinsic networks, we applied high-model-order independent component analysis (ICA) to the preprocessed data by using the GIFT-toolbox ${ }^{1}$ with the infomax algorithm implemented in Matlab (Calhoun et al., 2001). Data were decomposed into 75 spatial independent components (IC), correspondent with a framework for high-model-order decomposition (Abou Elseoud et al., 2011; Allen et al., 2011). High-model-order ICA approaches of about 70 components yield IC, which are in optimal accordance with known anatomical and functional segmentations (Damoiseaux et al., 2006; Kiviniemi et al., 2009; Smith et al., 2009). Data were concatenated and reduced by two-step principal component analysis (PCA), followed by independent component estimation

\footnotetext{
${ }^{1}$ http://icatb.sourceforge.net
} 
with the infomax-algorithm. We subsequently ran 40 ICAs (ICASSO) to ensure stability of the estimated components (Himberg et al., 2004). This results in a set of average group components which are then back reconstructed into single subject space, each represented by a spatial map of z-scores reflecting the within-network iFC and one associated time course of BOLD-signal fluctuations representative for this IC.

To select the IC reflecting networks of interest in an automated and objective way, we conducted multiple spatial regressions of 75 IC' spatial maps on T-maps of intrinsic connectivity networks (ICNs) as described in Allen et al. (2011). These T-maps were generated by the identical ICA approach as performed in the current study based on 603 healthy adolescents and adults and were made available online by the Medical Image Analysis Lab (MIALAB). ${ }^{2}$ For each ICN, the independent component with the largest correlation coefficient was chosen. According to our hypothesis, we restricted our selection to ICNs, which were characterized as part of either SN, DMN, or CEN (ICs 25, 34, 50, 53, 55, 60, 68 in Allen et al., 2011), resulting in a total of seven ICNs for further analysis.

To define outcome measures of inter-network iFC, we performed Pearson correlation analyses for all of these networks resulting in 21 correlations per participant. Pearson correlation coefficients were transformed into $z$-values via Fisher r-to-z transformation. Subsequently, we correlated these $\mathrm{z}$-values with the two measures of mindfulness ability across participants, respectively, and evaluated statistical significance of results via $t$-tests $(p<0.05$, Bonferroni corrected for 21 tests with corrected $p$-value $p<0.0024)$.

${ }^{2}$ http://mialab.mrn.org/data/hcp/RSN_HC_unthresholded_tmaps.nii
In order to test the specificity of the link between interiFC and mindfulness for neuro-cognitive key networks, we additionally selected three visual occipital networks from Allen et al. (2011): IC 46, 64 and 67 and performed the identical analysis, including computation of inter-iFC between these visual networks and associations with mindfulness scores. We chose occipital visual networks, since a previous study demonstrated intra-network connectivity changes in such a visual network after 8 week mindfulness training (Kilpatrick et al., 2011), suggesting that its inter-network connectivity might be linked with trait mindfulness, too. Furthermore, we performed a similar analysis for the inter-iFC between the visual and neuro-cognitive networks, respectively. That is we computed inter-iFC and its association with mindfulness scores.

\section{Results}

\section{Psychometric Assessment of Mindfulness}

Mindfulness was assessed using the MAAS and the FMI. Mean scores were $60.9(\mathrm{SD}=8.5)$ for the MAAS and $37.5(\mathrm{SD}=3.1)$ for the FMI. The FMI contained two outliers (based on the outlier labeling rule; Hoaglin and Iglewicz, 1987) that were excluded from all analyses pertaining to FMI scores. The correlation between the questionnaires was significant $(r=0.45, p<0.02)$.

\section{Intrinsic Networks and Inter-Network Connectivity}

Automated component selection revealed seven components of interest for each individual. Selected components matched previous results of $\mathrm{SN}, \mathrm{DMN}$, and CEN (Allen et al., 2011; Figure 1; Table 1; $p<0.05$, FWE-corrected). The SN was represented in two components, comprising



FIGURE 1 | Intrinsic networks of interest. Shown are spatial maps of one-sample $t$-tests (voxel wise family wise error corrected, $p<0.05$ ) Abbreviations: aDMN, anterior default mode network; pvDMN, posterior ventral default mode network; pdDMN, posterior dorsal default mode network; accSN cingular salience network; insSN, insula salience network; ICEN, left central executive network; rCEN, right central executive network. 
TABLE 1 | Intrinsic brain networks of interest.

\begin{tabular}{|c|c|c|c|c|c|c|c|}
\hline \multirow[t]{2}{*}{ Network } & \multirow[t]{2}{*}{ Region } & \multirow[t]{2}{*}{ Voxel: $t$-value } & \multirow[t]{2}{*}{$P$-value } & \multirow[t]{2}{*}{ Cluster size } & \multicolumn{3}{|c|}{ Peak MNI-coordinates } \\
\hline & & & & & $x$ & $y$ & $\mathbf{z}$ \\
\hline \multirow[t]{4}{*}{ aDMN } & Left anterior cingulate cortex & 30.7 & $<0.01$ & 2047 & -6 & 53 & 1 \\
\hline & Left middle orbital gyrus & 30.6 & $<0.01$ & & -6 & 47 & -8 \\
\hline & Right anterior cingulate cortex & 29 & $<0.01$ & & 6 & 47 & 7 \\
\hline & Left posterior cingulate cortex & 11.6 & $<0.01$ & 87 & 0 & -52 & 28 \\
\hline \multirow[t]{4}{*}{ pvDMN } & Right precuneus & 39.98 & $<0.01$ & 2632 & 6 & 61 & 28 \\
\hline & Left posterior cingulate cortex & 28.34 & $<0.01$ & & -6 & -52 & 28 \\
\hline & Left precuneus & 34.4 & $<0.01$ & & -6 & -61 & 34 \\
\hline & Right middle cingulate cortex & 28.7 & $<0.01$ & & 12 & -49 & 34 \\
\hline \multirow[t]{3}{*}{ pdDMN } & Left precuneus & 27.3 & $<0.01$ & 1275 & -12 & -67 & 34 \\
\hline & Right precuneus & 26.3 & $<0.01$ & & 9 & -70 & 37 \\
\hline & Left cuneus & 24.8 & $<0.01$ & & -6 & -76 & 34 \\
\hline \multirow[t]{2}{*}{$\operatorname{accSN}$} & Right middle cingulate cortex & 27.1 & $<0.01$ & 2714 & 9 & 26 & 31 \\
\hline & Left anterior cingulate cortex & 26.7 & $<0.01$ & & -6 & 14 & 28 \\
\hline \multirow[t]{5}{*}{ insSN } & Right insular lobe & 34.2 & $<0.01$ & 891 & 45 & 14 & 1 \\
\hline & Left insular lobe & 25.8 & $<0.01$ & 813 & -36 & 17 & 1 \\
\hline & Left inferior frontal gyrus & 18.8 & $<0.01$ & & -39 & 14 & -5 \\
\hline & Right middle cingulate cortex & 11.5 & $<0.01$ & 280 & 9 & 11 & 40 \\
\hline & Left middle cingulate cortex & 9.7 & $<0.01$ & & 0 & 26 & 34 \\
\hline \multirow[t]{6}{*}{ ICEN } & Left middle frontal gyrus & 26.2 & $<0.01$ & 1808 & -36 & 56 & 7 \\
\hline & Left superior frontal gyrus & 19.9 & $<0.01$ & & -24 & 13 & 63 \\
\hline & Left inferior parietal lobule & 18.9 & $<0.01$ & & -54 & -49 & 43 \\
\hline & Left angular gyrus & 26.9 & $<0.01$ & 1342 & -42 & -58 & 46 \\
\hline & Left inferior temporal gyrus & 19.5 & $<0.01$ & 104 & -54 & -49 & -8 \\
\hline & Left middle cingulate cortex & 10.0 & $<0.01$ & & -3 & -34 & 43 \\
\hline \multirow[t]{8}{*}{ rCEN } & Right inferior parietal lobule & 34 & $<0.01$ & 1027 & 54 & -58 & 40 \\
\hline & Right angular gyrus & 32.9 & $<0.01$ & & 51 & -58 & 31 \\
\hline & Right middle frontal gyrus & 16.8 & $<0.01$ & 603 & 33 & 17 & 52 \\
\hline & Right superior frontal gyrus & 13.5 & $<0.01$ & & 21 & 26 & 55 \\
\hline & Left angular gyrus & 17.2 & $<0.01$ & 338 & -42 & -58 & 34 \\
\hline & Left inferior parietal lobule & 14.5 & $<0.01$ & & -48 & -55 & 46 \\
\hline & Right precuneus & 13.5 & $<0.01$ & 216 & 6 & -58 & 37 \\
\hline & Right middle cingulate cortex & 13.5 & $<0.01$ & & 6 & -46 & 34 \\
\hline
\end{tabular}

Shown are results of one-sample t-tests of resting state brain intrinsic brain networks after independent component analysis with 75 components (voxel wise family wise error correction for multiple comparisons, $p<0.05)$. Abbreviations: DMN, default mode network; SN, salience network; CEN, central executive network; aDMN, anterior default mode network; pvDMN, posterior ventral default mode network; pdDMN, posterior dorsal default mode network; accSN, cingular salience network; insSN, insula salience network; ICEN, left central executive network; rCEN, right central executive network.

ACC (accSN) and bilateral anterior insula (insSN). $\mathrm{DMN}$ was represented in three components [anterior DMN (aDMN; medial prefrontal cortex), posterior-ventral DMN (pvDMN; posterior cingulate cortex, PCC) and posteriordorsal DMN (pdDMN; precuneus)]. CEN was represented in two components, comprising two lateralized fronto-parietal networks corresponding to left (lCEN; left middle and superior frontal cortex, and inferior parietal lobule), and right CEN (rCEN; right middle and superior frontal gyri, right angular gyrus, and right inferior parietal lobule). The three visual control networks were located in medial and lateral occipital cortex and matched well with the templates in Allen et al. (2011; Figure 2).

From the seven intrinsic networks of interest and the three control networks, we extracted the network time courses and defined inter-iFC via Pearson's correlation (see Table 2 for mean correlation coefficients of networks of interest and Table 4 for visual control networks).

\section{Association between Inter-iFC and Mindfulness Scores}

After Fisher r-to-z transformation, we correlated $\mathrm{z}$-scores of inter-iFC with the mindfulness scores of both FMI and MAAS (Pearson correlation, $p<0.05$, Bonferroni corrected for 21 comparisons, corrected threshold $p<0.0024$; Figures 3, 4; Table 3). We found significant negative correlations between FMI and inter-iFC of insSN and pvDMN $(r=-0.60, p<0.002)$. In addition, inter-iFC between the aDMN and the pdDMN was significantly negatively correlated with the MAAS scores $(r=-0.65, p<0.001)$. At border significance (i.e., $p<0.05$, but not surviving correction for multiple testing), we found negative associations between MAAS and inter-iFC of aDMN and lCEN $(r=-0.40, p<0.045)$, FMI and inter-iFC of insSN and $\operatorname{aDMN}(r=-0.53$, significance $p<0.008)$, as well as FMI and inter-iFC between accSN and lCEN $(r=-0.45$, $p<0.03)$.

To evaluate the influence of sex on the association between mindfulness and inter-network connectivity, we re-analyzed the 
TABLE 2 | Functional connectivity between intrinsic networks.

\begin{tabular}{|c|c|c|c|c|c|c|c|}
\hline Network & insSN & pdDMN & pvDMN & aDMN & $\operatorname{accSN}$ & rCEN & ICEN \\
\hline insSN & 1.00 & 0.08 & -0.14 & 0.08 & 0.14 & -0.09 & -0.05 \\
\hline pdDMN & 0.08 & 1.00 & 0.29 & 0.25 & 0.06 & 0.23 & 0.24 \\
\hline pvDMN & -0.14 & 0.29 & 1.00 & 0.33 & -0.17 & 0.37 & 0.24 \\
\hline aDMN & 0.08 & 0.25 & 0.33 & 1.00 & 0.17 & 0.29 & 0.15 \\
\hline $\operatorname{accSN}$ & 0.14 & 0.06 & -0.17 & 0.17 & 1.00 & 0.08 & 0.01 \\
\hline rCEN & -0.09 & 0.23 & 0.37 & 0.29 & 0.08 & 1.00 & 0.25 \\
\hline ICEN & -0.05 & 0.24 & 0.24 & 0.15 & 0.01 & 0.25 & 1.00 \\
\hline
\end{tabular}

Shown are mean Pearson-correlation coefficients for each pair of networks of interest. Abbreviations: aDMN, anterior default mode network; pvDMN, posterior ventral default mode network; pdDMN, posterior dorsal default mode network; accSN, cingular salience network; insSN, insula salience network; ICEN, left central executive network; rCEN, right central executive network.

dependence between inter-iFC and MAAS and FMI, respectively, by the use of partial correlation analysis with sex as additional control variable. In partial correlation analysis, the dependence between two variables (i.e., inter-iFC and mindfulness score) is evaluated while controlling for the influence of further variables (i.e., sex) on both variables of interest, respectively. While almost all results concerning the link between interiFC and mindfulness scores changed only marginally, merely the association between MAAS and inter-iFC of aDMN and lCEN, which was at-trend $(p=0.05)$ in the analysis without control for sex, lost its trend to significance. This finding indicates that the observed link between mindfulness and interiFC between $\mathrm{SN}$ and DMN is largely independent from sex differences.

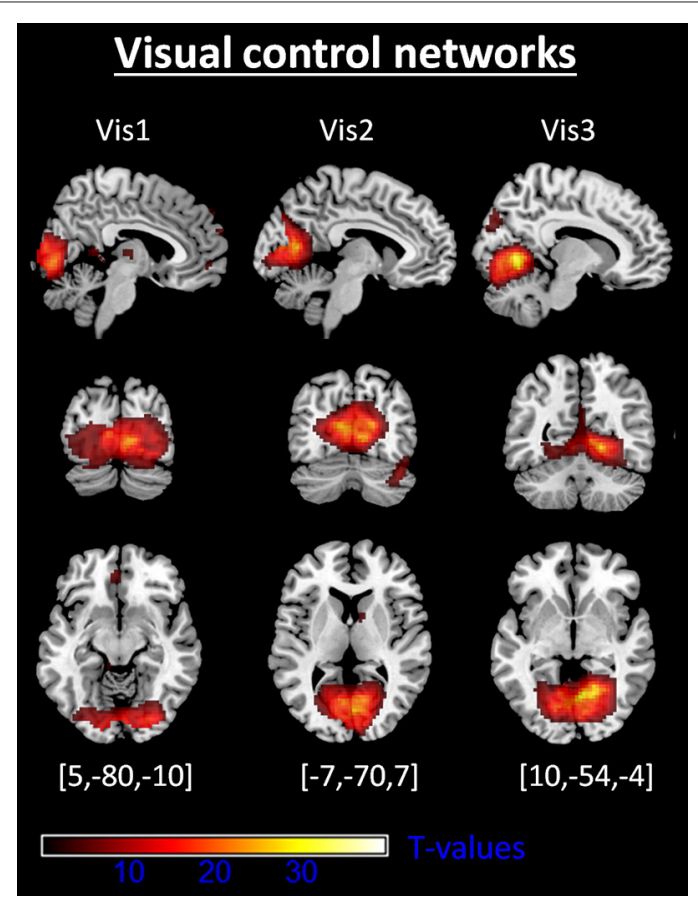

FIGURE 2 | Intrinsic visual control networks. Shown are spatial maps of one-sample $t$-tests (voxel wise family wise error corrected, $p<0.05$ ). Abbreviations: Vis1, visual network 1; Vis2, visual network 2; Vis3, visual network 3. coordinates are given with respect to Montreal Neurological Institute standard space.
For the association between mindfulness scores and inter-iFC among visual control networks, we found no significant correlation (Table 4). The analysis of the association between mindfulness scores and inter-iFC between visual and neuro-cognitive networks of interest yielded one at trend result (Table 5). The correlation between FMI and the connectivity between the anterior DMN and a secondary visual network (reflecting IC 67 component of Allen et al. (2011) and covering mainly the lingual gyrus) had a Pearson's coefficient of $r=-0.53$ with a $p$-value of 0.01 . This $p$-value did not survive correction for multiple testing via Bonferroni for corrected threshold of $p<0.0024$.

\section{Discussion}

The present study investigated the association between mindfulness and functional connectivity of intrinsic brain activity among three central neurocognitive networks (inter-iFC): the SN, DMN and CEN. Inter-iFC between DMN and $\mathrm{SN}$-and inter-iFC between $\mathrm{SN}$ and CEN at trend-was correlated with mindfulness scores. Results suggest that mindfulness is significantly associated with ongoing network interactions of central neurocognitive networks.

\section{Relationship between Mindfulness and Inter-Network Connectivity}

We found negative correlations of mindfulness ability and inter-iFC among SN, DMN and CEN (Figures 3, 4; Table 3). These correlations were significant for the negative inter-iFC between insSN and pvDMN. This result was specific for neuro-cognitive key networks, since control analysis for visual networks inter-network connectivity did not yield significant results (Table 5). Furthermore it was independent from sex differences, for which we controlled in additional control analyses. The increase in anti-correlation between the insSN and pvDMN for more mindful individuals replicates previous findings (Kilpatrick et al., 2011). Kilpatrick et al. (2011) compared intrinsic brain networks in participants of an 8 week course of mindfulness stress reduction (MBSR) to waitlist controls during a mindful resting state. After training in MBSR, participants showed increased anti-correlation between a region within the cuneus (part of the pvDMN) and their equivalent of the SN (Kilpatrick et al., 2011). In another study, 

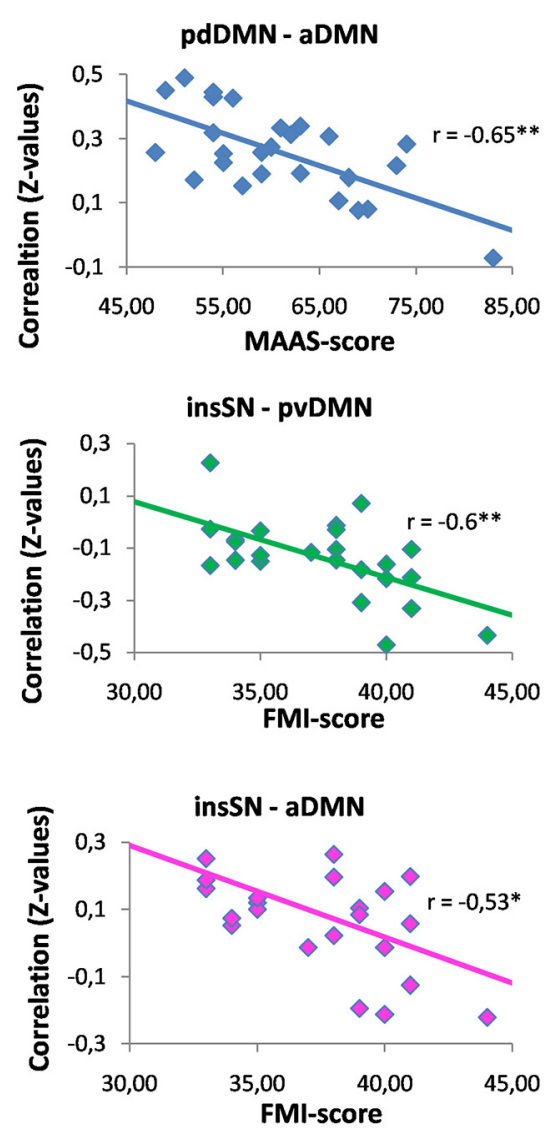

FIGURE 3 | Correlations between mindfulness ability and inter-network functional connectivity. Abbreviations: MAAS, Mindful Attention and Awareness Scale; FMI, Freiburg Mindfulness Inventory; aDMN, anterior default mode network; pvDMN, posterior ventral default mode network; pdDMN,

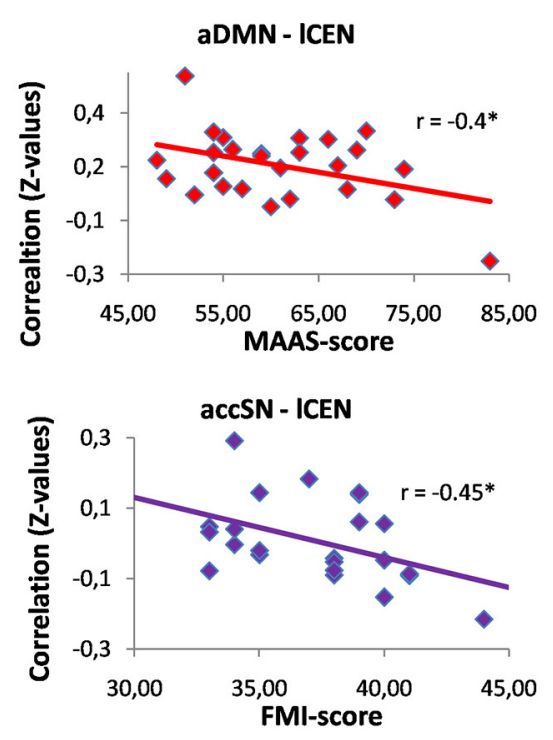

posterior dorsal default mode network; accSN, cingular salience network: insSN, insula salience network; ICEN, left central executive network; rCEN, right central executive network. ${ }^{*} p<0.05$; ${ }^{* *}$ significant with Bonferroni correction for multiple comparisons.
Hasenkamp and Barsalou (2012) performed a whole brain resting state FC analysis of a network comprising bilateral posterior cingulate cortex (PCC) and ventro-medial prefrontal cortex (vmPFC) regions of interest, representing the DMN, and compared this between high and low practice meditators. Supporting the present study, they found a decrease in correlation between the hubs of the DMN and the insSN, in high practiced meditators (Hasenkamp and Barsalou, 2012). The SN has been hypothesized to be involved in the detection and evaluation of motivationally salient stimuli, i.e., stimuli with relevance for the organism (Seeley et al., 2007) and in controlling interactions between the DMN and CEN (Sridharan et al., 2008), while the DMN is involved in memory, selfrelated, and social processing (for a review, see Buckner et al., 2008). Furthermore, the $\mathrm{DMN}$ has been shown to be activated during mind wandering (Mason et al., 2007; Hasenkamp et al., 2012). Anti-correlation could be interpreted as a clearer distinction between the networks which might result in better effective connectivity (Clare Kelly et al., 2008; Deco et al., 2009; Lewis et al., 2009). Following this line of thought, an increased anti-correlation between insSN and the pvDMN might indicate improved sensitivity to mind wandering in more mindful individuals. This anti-correlationbased connectivity between the insSN and pvDMN was particularly related to the FMI, which is focused on measuring the accepting stance towards all experience that is inherent to mindfulness (Walach et al., 2006). The pvDMN included the PCC, which is active during personal evaluations (WhitfieldGabrieli et al., 2011) and emotional processing (Kober et al., 2008), which has been hypothesized to be related to the meditative experience (Brewer and Garrison, 2014). Together with the insSN's involvement in directing attention to salient stimuli (Seeley et al., 2007), stronger negative correlation between pvDMN and insSN might indicate a less evaluative stance during rest towards experience, which would fit well with the topic of the FMI measuring acceptance (Walach et al., 2006).

Interestingly, participants of the studies cited above (Kilpatrick et al., 2011; Hasenkamp and Barsalou, 2012) had considerable more experience with meditation than the participants of the present study, but show a comparable pattern of changes in connectivity. It seems thus, that changes in 
TABLE 3 | Pearson's correlation coefficients between inter-network intrinsic functional connectivity (inter-iFC) and mindfulness ability.

\begin{tabular}{|c|c|c|c|c|c|}
\hline & rCEN/ICEN & aDMN/ICEN & aDMN/rCEN & aDMN/accSN & pvDMN/ICEN \\
\hline$r$-value FMI & 0.05 & 0.29 & -0.06 & 0.02 & 0.13 \\
\hline$P$-value & 0.82 & 0.16 & 0.79 & 0.94 & 0.54 \\
\hline$r$-value MAAS & -0.12 & -0.40 & -0.18 & 0.02 & -0.33 \\
\hline \multirow[t]{2}{*}{$P$-value } & 0.55 & $0.05^{*}$ & 0.39 & 0.92 & 0.10 \\
\hline & pdDMN/ICEN & pdDMN/rCEN & pdDMN/aDMN & pdDMN/pvDMN & pdDMN/accSN \\
\hline$r$-value FMI & 0.13 & 0.01 & -0.36 & -0.16 & 0.14 \\
\hline$P$-value & 0.53 & 0.98 & 0.09 & 0.45 & 0.50 \\
\hline$r$-value MAAS & 0.24 & -0.13 & -0.65 & -0.10 & 0.03 \\
\hline \multirow[t]{2}{*}{$P$-value } & 0.24 & 0.51 & $<0.01^{* *}$ & 0.63 & 0.87 \\
\hline & pvDMN/rCEN & pvDMN/aDMN & pvDMN/accSN & accSN/ICEN & $\operatorname{accSN} / \mathrm{rCEN}$ \\
\hline r-value FMI & -0.05 & -0.09 & 0.11 & -0.45 & -0.14 \\
\hline$P$-value & 0.80 & 0.69 & 0.61 & $0.03^{*}$ & 0.53 \\
\hline$r$-value MAAS & -0.09 & -0.30 & 0.04 & 0.06 & -0.11 \\
\hline \multirow[t]{2}{*}{$P$-value } & 0.66 & 0.14 & 0.83 & 0.79 & 0.59 \\
\hline & insSN/ICEN & insSN/rCEN & insSN/aDMN & insSN/pvDMN & insSN/accSN \\
\hline$r$-value FMI & -0.04 & -0.34 & -0.53 & -0.60 & -0.02 \\
\hline$P$-value & 0.86 & 0.10 & $0.01^{*}$ & $<0.01^{* *}$ & 0.94 \\
\hline$r$-value MAAS & 0.33 & 0.08 & -0.13 & -0.14 & 0.02 \\
\hline \multirow[t]{2}{*}{$P$-value } & 0.09 & 0.69 & 0.53 & 0.49 & 0.93 \\
\hline & insSN/pdDMN & & & & \\
\hline$r$-value FMI & -0.09 & & & & \\
\hline$P$-value & 0.67 & & & & \\
\hline$r$-value MAAS & 0.30 & & & & \\
\hline$P$-value & 0.14 & & & & \\
\hline
\end{tabular}

Abbreviations: MAAS, Mindful Attention and Awareness Scale; FMI, Freiburg Mindfulness Inventory; aDMN, anterior default mode network; pvDMN, posterior ventral default mode network; pdDMN, posterior dorsal default mode network; accSN, cingular salience network; insSN, insula salience network; ICEN, left central executive network; rCEN, right central executive network. ${ }^{*} p<0.05$; ** significant with Bonferroni correction for multiple comparisons for 21 multiple comparisons (corrected threshold $p=0.0024$ ).

connectivity between the SN and the DMN are among the first to become apparent during mindfulness training that could be extended by changes in connectivity of e.g., task positive or attentional networks.

In addition to the above mentioned results, we found several results that were significant only at trend level. The connection of the aDMN and the lCEN being sensitive to mindfulness may confirm results found previously for experienced meditators (Hasenkamp and Barsalou, 2012; Taylor et al., 2013) although these studies found an increase in connectivity rather than a reduction for experienced meditators. The CEN is thought

TABLE 4 | Control analysis: Inter-network connectivity and associations with mindfulness of visual networks.

\begin{tabular}{lccr}
\hline Network & Vis1/Vis2 & Vis1/Vis3 & Vis2/Vis3 \\
\hline Inter-iFC & 0.27 & 0.43 & 0.38 \\
Correlation with FMI & -0.34 & -0.33 & -0.38 \\
$p$-value & 0.1 & 0.11 & 0.07 \\
Correlation with MAAS & -0.18 & -0.12 & -0.09 \\
$p$-value & 0.38 & 0.57 & 0.67 \\
\hline
\end{tabular}

Shown are mean Pearson-correlation coefficients for each pair of visual control networks and below corresponding correlations of inter-iFC and mindfulness scores. Abbreviations: Vis1, visual network 1; Vis2, visual network 2; Vis3, visual network 3; FMI, Freiburg Mindfulness Inventory; MAAS, Mindful Attention and Awareness Scale. to be involved in the redirection of attention (Corbetta and Shulman, 2002), while the aDMN, specifically the ventromedial PFC is involved in self-related processing (e.g., AndrewsHanna et al., 2010). The present data might suggest that more mindful individuals may have a lower correlation between these networks, which could indicate an increased switching of attention away from self-related towards e.g., more sensory focused processing.

Moreover, we found a relationship of mindfulness scores and connectivity of the lCEN and the accSN, which had not been investigated previously. The accSN has been associated with processing of emotions, cognition and action inhibition (Smith et al., 2009). Speculating towards a reduced connectivity between the lCEN and accSN in more mindful individuals, it might be possible that this emphasizes conscious attentional processing over emotional value based evaluation of stimuli. Together, the present data show that connectivity in resting state networks may be sensitive for mindfulness effects. Concerning the direction of mindfulness effect (i.e., more or less inter-iFC), there are a number of inconsistencies about certain connections and the directionality in the literature and consequently when comparing our findings with those of previous studies. Possible explanations for these irregularities could be factors related to study design that can influence resting state connectivity. Activations during tasks performed immediately before a resting sate scan have been shown to carry over into resting state 
TABLE 5 | Control analysis: inter-network connectivity between visual networks and neuro-cognitive key networks and associations with mindfulness ability.

\begin{tabular}{|c|c|c|c|c|c|c|c|}
\hline Network & insSN & pdDMN & pvDMN & aDMN & $\operatorname{accSN}$ & ICEN & rCEN \\
\hline Visual 1 & 0.04 & 0.30 & 0.17 & 0.13 & -0.08 & 0.15 & -0.05 \\
\hline Correlation with FMl & -0.07 & -0.12 & 0.02 & -0.36 & 0.35 & 0.16 & 0.31 \\
\hline$p$-value & 0.75 & 0.57 & 0.93 & 0.08 & 0.09 & 0.45 & 0.14 \\
\hline Correlation with MAAS & 0.21 & 0.00 & -0.22 & -0.09 & 0.28 & -0.13 & 0.10 \\
\hline$p$-value & 0.31 & 0.99 & 0.27 & 0.66 & 0.16 & 0.51 & 0.64 \\
\hline Visual 2 & 0.05 & 0.29 & 0.23 & 0.09 & -0.18 & 0.11 & $>-0.01$ \\
\hline Correlation with FMI & -0.24 & -0.11 & -0.27 & 0.02 & 0.04 & 0.10 & 0.20 \\
\hline$p$-value & 0.25 & 0.61 & 0.20 & 0.94 & 0.84 & 0.64 & 0.36 \\
\hline Correlation with MAAS & 0.14 & 0.00 & -0.23 & -0.27 & -0.22 & 0.35 & -0.18 \\
\hline$p$-value & 0.51 & 0.99 & 0.26 & 0.17 & 0.28 & 0.08 & 0.37 \\
\hline Visual 3 & 0.10 & 0.23 & 0.12 & $<0.01$ & -0.21 & 0.04 & -0.07 \\
\hline Correlation with FMI & -0.17 & -0.09 & -0.14 & -0.53 & 0.28 & 0.28 & 0.06 \\
\hline$p$-value & 0.43 & 0.69 & 0.52 & $0.01^{*}$ & 0.18 & 0.18 & 0.79 \\
\hline Correlation with MAAS & 0.13 & 0.08 & -0.18 & -0.16 & 0.09 & 0.15 & $<0.01$ \\
\hline$p$-value & 0.53 & 0.69 & 0.37 & 0.43 & 0.65 & 0.47 & 0.98 \\
\hline
\end{tabular}

Shown are mean Pearson-correlation coefficients for each pair of networks of interest and three control networks. In addition, correlations and p-values of the respective inter network connectivity and mindfulness scores are shown. Abbreviations: aDMN, anterior default mode network; pvDMN, posterior ventral default mode network; pdDMN, posterior dorsal default mode network; accSN, cingular salience network; insSN, insula salience network; ICEN, left central executive network; rCEN, right central executive network. Visual 1, visual network; Visual 2, visual network 2; Visual 3, visual network 3; * $p$-value significant without bonferroni correction (cutoff $p=0.0024$ ).

connectivity (Tambini et al., 2010). This might e.g., occur if meditation is performed or trained immediately before the resting state scan. Researchers should consider an appropriate break between task or training and rest scans. Similarly, we

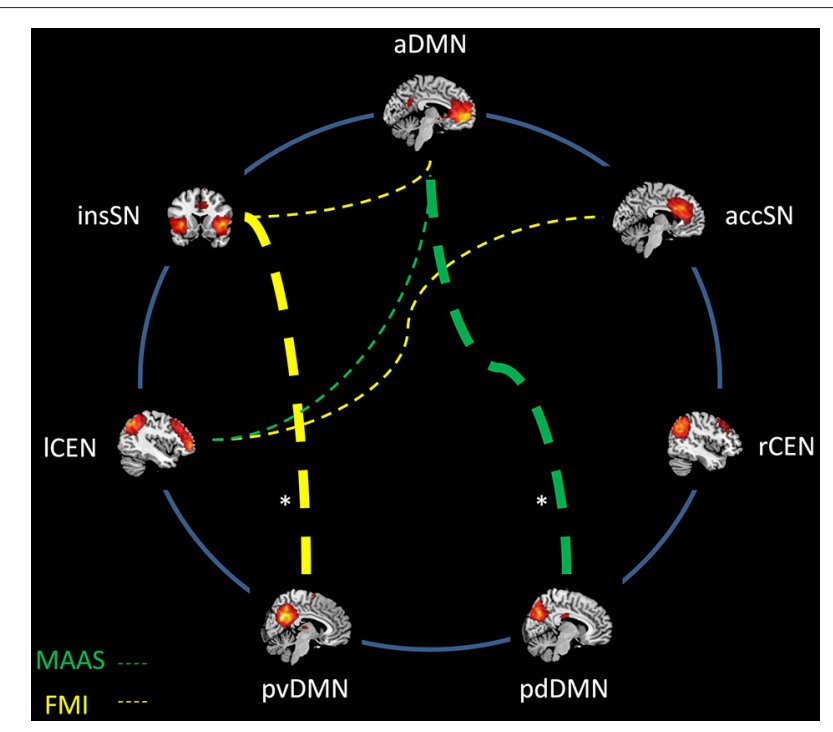

FIGURE 4 | Association between mindfulness ability and inter-network intrinsic functional connectivity (iFC). Networks are represented by spatial maps of one-sample $t$-tests (voxel wise family wise error corrected, $p<0.05$ ). Dashed lines indicate negative correlations between mindfulness and connectivity strength. Abbreviations: MAAS, Mindful Attention and Awareness Scale; FMI, Freiburg Mindfulness Inventory; aDMN, anterior default mode network; pvDMN, posterior ventral default mode network; pdDMN, posterior dorsal default mode network; accSN, cingular salience network; insSN, insula salience network; ICEN, left central executive network; rCEN, right central executive network. *significant with Bonferroni correction for multiple comparisons. cannot control for what participants actually do during the resting state scan. Especially in meditation research this aspect is critical as meditation itself is performed under conditions very similar to resting and some individuals might by default engage in focused attention or other meditation during rest. This aspect is seldom discussed and should receive additional attention. Future studies should carefully instruct participants before performing a resting state scan making sure participants do not enter a meditation state.

\section{Connectivity Between the Anterior and Posterior DMN}

The present study showed that the connectivity of the anterior and the posterior parts of the DMN was sensitive to mindfulness ability (Figure 3). The DMN has most strongly been in the focus of mindfulness research and a number of studies have reported connectivity changes related to mindfulness (Brewer et al., 2011; Jang et al., 2011; Kilpatrick et al., 2011; Hasenkamp and Barsalou, 2012; Shaurya Prakash et al., 2013; Taylor et al., 2013). The present study showed decreased connectivity in individuals with higher mindfulness ability between the aDMN and pdDMN. This result is in accordance with the results by Hasenkamp and Barsalou (2012) who found that a region in $\mathrm{mPFC} / \mathrm{ACC}$ showed decreased connectivity with the PCC, the main hub of the pDMN, in mindfulness experts compared to novices. However, this result seems to contradict two previous results, which showed increased connectivity in more mindful individuals (Shaurya Prakash et al., 2013) and in experienced meditators (Jang et al., 2011). Yet, while one of these studies focused on elderly participants (Shaurya Prakash et al., 2013) the other used a region of interest approach to identify the DMN, which does not differentiate between posterior and anterior DMNs (Jang et al., 2011). These differences in study design might explain the difference in the results. In addition, other 
studies did not find any relationship for the connection of the anterior and posterior parts of the DMN with mindfulness (Kilpatrick et al., 2011; Taylor et al., 2013). The main hubs of the DMN are the precuneus/PCC (posterior DMN) and the vmPFC/ACC region (anterior DMN). The precuneus, is involved in the affective relevance of a given stimulus and is a critical structure for conscious processing (for a review, see Vogt and Laureys, 2005) while the vmPFC is involved in self-referential processing (Andrews-Hanna et al., 2010). A decreased connectivity of the aDMN and pDMN might indicate that more mindful individuals interpret the affective relevance of a given stimulus as less self-related. This is also supported by the association of the MAAS questionnaire with this connectivity. The MAAS focuses on measuring the ability to consciously perceive the present moment (Brown and Ryan, 2003). This present moment experience has been associated with a deactivation of the PCC/Precuneus (Garrison et al., 2013) area and with activations in dorso medial (Hölzel et al., 2007; Dickenson et al., 2013) and lateral prefrontal regions (Brefczynski-Lewis et al., 2007). Thus, this would speak for a reduced synchrony of the antDMN and the $\mathrm{pDMN}$ regions during this experience, which could transition into a stronger decoupling of these parts of the DMN network in more mindful individuals during rest. Instead, these regions may be coupled more strongly to either lateral parietal or DLPFC in expert meditators. e.g., Brewer et al. (2011) found increased connectivity between the PCC, dorsal ACC and DLPFC in participants with more meditation experience both during rest and during different kinds of meditation. The authors interpreted these results as an at baseline increased connectivity and activity of task positive control regions together with reduced activation of the DMN in experienced meditators regardless of condition. Other authors have argued for a coactivation of the aDMN together with inferior parietal regions during rest, which might reduce distractibility by mind wandering in experienced meditators (Hasenkamp and Barsalou, 2012). Our data are more in accordance with the model by Hasenkamp and Barsalou (2012), which suggests a critical interplay between medial DMN and lateral CEN for engaging attention on present experience. Instead of being engaged in mind-wandering which results in activation of the anterior and posterior DMN (Mason et al., 2007), regions in the dmPFC might be responsible for focusing attention back to present experience likely reflected by stronger anti-correlated coupling between CEN and DMN. Future studies will have to further clarify the directionality of connectivity between the anterior DMN, posterior DMN, and attention-relevant regions of frontal and parietal CEN and how this is related to meditation experience and mindfulness disposition.

\section{Limitations}

To evaluate the key findings of our study, some limitations have to be considered. Firstly, our approach to study the relevance of inter-iFC in neuro-cognitive key networks for mindfulness is a correlation-based approach (i.e., we linked mindfulness scores with inter-iFC via linear correlation). Whether increased negative iFC between SN and DMN, which we demonstrated to correlate with mindfulness ability, is causal for the variability in mindfulness ability is not addressable by a correlation-based approach. To address such causal link between inter-iFC and mindfulness, controlled longitudinal training studies of mindfulness practice are necessary. In such studies, a controlled change in mindfulness ability can be linked with changes in inter-iFC of neuro-cognitive key networks. Secondly, given the wide range of functional domains, in which $\mathrm{SN}, \mathrm{CEN}$, and DMN are involved, it is hard to specify the behavioral implications of inter-iFC variability for mindfulness (i.e., which functional aspect of neuro-cognitive networks is relevant for mindfulness). Future studies combining resting-state and task fMRI with mindfulness might be helpful, if tasks are included that are part of mindfulness practice such as e.g., "focused attention to breathing."

\section{Mindfulness as a Counterweight of Psychopathology}

It is striking that mindfulness impacts the connectivity of three resting state networks (DMN, CEN, and SN) that have been shown to play a critical role in various psychopathologies (Menon, 2011). In detail, it has been hypothesized that the SN acts to control switching between DMN and CEN: depending on the demands of the task at hand the $\mathrm{SN}$ is hypothesized to balance activation in the other two networks (Sridharan et al., 2008; Menon and Uddin, 2010). Given that mindfulness has been shown to reduce psychopathological symptoms (Baer, 2003; Aldao et al., 2010; Hofmann et al., 2010), affecting the inter-iFC might be one possible pathway for beneficial effects of mindfulness in psychotherapy.

\section{Conclusion}

Mindfulness is correlated with the inter-iFC of subnetworks of the DMN and SN. Specifically, more mindful individuals show a decreased correlation between the aDMN and pdDMN and stronger negative correlation between the insSN and pvDMN.

\section{Funding}

This work was supported by the Elitenetzwerk Bayern (through the Ludwigs-Maximilians Universität and the Klinikum rechts der Isar, Technischen Universität München) for $\mathrm{AD}$; the German Federal Ministry of Education and Research [BMBF 01EV0710 to A.M.W., BMBF 01ER0803 to CS], the Kommission für Klinische Forschung, Technische Universität München [KKF 8765162 to CS] and the Technische Universität München within the funding program Open Access Publishing.

\section{Acknowledgments}

We thank our colleague Dr. Christine Preibisch for her help with the imaging procedures. 


\section{References}

Abou Elseoud, A., Littow, H., Remes, J., Starck, T., Nikkinen, J., Nissilä, J., et al. (2011). Group-ICA model order highlights patterns of functional brain connectivity. Front. Syst. Neurosci. 5:37. doi: 10.3389/fnsys.2011.00037

Aldao, A., Nolen-Hoeksema, S., and Schweizer, S. (2010). Emotion-regulation strategies across psychopathology: a meta-analytic review. Clin. Psychol. Rev. 30, 217-237. doi: 10.1016/j.cpr.2009.11.004

Allen, E. A., Erhardt, E. B., Damaraju, E., Gruner, W., Segall, J. M., Silva, R. F., et al. (2011). A baseline for the multivariate comparison of resting-state networks. Front. Syst. Neurosci. 5:2. doi: 10.3389/fnsys.2011.00002

Andrews-Hanna, J. R., Reidler, J. S., Sepulcre, J., Poulin, R., and Buckner, R. L. (2010). Functional-anatomic fractionation of the brain's default network. Neuron 65, 550-562. doi: 10.1016/j.neuron.2010.02.005

Baer, R. A. (2003). Mindfulness training as a clinical intervention: a conceptual and empirical review. Clin. Psychol. Sci. Pract. 10, 125-143. doi: 10.1093/clipsy. bpg015

Berkes, P., Orbán, G., Lengyel, M., and Fiser, J. (2011). Spontaneous cortical activity reveals hallmarks of an optimal internal model of the environment. Science 331, 83-88. doi: 10.1126/science. 1195870

Berkovich-Ohana, A., Glicksohn, J., and Goldstein, A. (2014). Studying the default mode and its mindfulness-induced changes using EEG functional connectivity. Soc. Cogn. Affect. Neurosci. 9, 1616-1624. doi: 10.1093/scan/nst153

Bishop, S., Lau, M., Shapiro, S., Carlson, L., Anderson, N. D., Carmody, J., et al. (2004). Mindfulness: a proposed operational definition. Clin. Psychol. Sci. Pract. 11, 230-241. doi: 10.1093/clipsy.bph077

Brefczynski-Lewis, J. A., Lutz, A., Schaefer, H. S., Levinson, D. B., and Davidson, R. J. (2007). Neural correlates of attentional expertise in long-term meditation practitioners. Proc. Natl. Acad. Sci. U S A 104, 11483-11488. doi: 10.1073/pnas. 0606552104

Brewer, J. A., and Garrison, K. A. (2014). The posterior cingulate cortex as a plausible mechanistic target of meditation: findings from neuroimaging. Ann. N Y Acad. Sci. 1307, 19-27. doi: 10.1111/nyas.12246

Brewer, J. A., Worhunsky, P. D., Gray, J. R., Tang, Y.-Y., Weber, J., and Kober, H. (2011). Meditation experience is associated with differences in default mode network activity and connectivity. Proc. Natl. Acad. Sci. U S A 108, 20254-20259. doi: 10.1073/pnas.1112029108

Brown, K. W., and Ryan, R. M. (2003). The benefits of being present: mindfulness and its role in psychological well-being. J. Pers. Soc. Psychol. 84, 822-848. doi: 10.1037/0022-3514.84.4.822

Buckner, R. L., Andrews-Hanna, J. R., and Schacter, D. L. (2008). "The brain's default network - anatomy, function and relevance to disease," in Year in Cognitive Neuroscience 2008, eds A. Kingstone, and M. B. Miller (Oxford: Blackwell Publishing), 1-38.

Calhoun, V. D., Adali, T., Pearlson, G. D., and Pekar, J. J. (2001). A method for making group inferences from functional MRI data using independent component analysis. Hum. Brain Mapp. 14, 140-151. doi: 10.1002/hbm.1048

Carmody, J. (2009). Evolving conceptions of mindfulness in clinical settings. J. Cogn. Psychother. 23, 270-280. doi: 10.1891/0889-8391.23.3.270

Clare Kelly, A. M., Uddin, L. Q., Biswal, B. B., Castellanos, F. X., and Milham, M. P. (2008). Competition between functional brain networks mediates behavioral variability. Neuroimage 39, 527-537. doi: 10.1016/j.neuroimage.2007.08.008

Corbetta, M., and Shulman, G. L. (2002). Control of goal-directed and stimulusdriven attention in the brain. Nat. Rev. Neurosci. 3, 201-215. doi: 10. 1038/nrn755

Creswell, J. D., Way, B. M., Eisenberger, N. I., and Lieberman, M. D. (2007). Neural correlates of dispositional mindfulness during affect labeling. Psychosom. Med. 69, 560-565. doi: 10.1097/psy.0b013e3180f6171f

Damoiseaux, J. S., Rombouts, S. A. R. B., Barkhof, F., Scheltens, P., Stam, C. J., Smith, S. M., et al. (2006). Consistent resting-state networks across healthy subjects. Proc. Natl. Acad. Sci. U S A 103, 13848-13853. doi: 10.1073/pnas. 0601417103

Deco, G., Jirsa, V., McIntosh, A. R., Sporns, O., and Kötter, R. (2009). Key role of coupling, delay and noise in resting brain fluctuations. Proc. Natl. Acad. Sci. U S A 106, 10302-10307. doi: 10.1073/pnas.0906701106

Dickenson, J., Berkman, E. T., Arch, J., and Lieberman, M. D. (2013). Neural correlates of focused attention during a brief mindfulness induction. Soc. Cogn. Affect. Neurosci. 8, 40-47. doi: 10.1093/scan/nss030
Fox, M. D., and Raichle, M. E. (2007). Spontaneous fluctuations in brain activity observed with functional magnetic resonance imaging. Nat. Rev. Neurosci. 8, 700-711. doi: 10.1038/nrn2201

Frewen, P. A., Dozois, D. J. A., Neufeld, R. W. J., Lane, R. D., Densmore, M., Stevens, T. K., et al. (2010). Individual differences in trait mindfulness predict dorsomedial prefrontal and amygdala response during emotional imagery: an fMRI study. Pers. Individ. Dif. 49, 479-484. doi: 10.1016/j.paid.2010.05.008

Froeliger, B., Garland, E. L., Kozink, R. V., Modlin, L. A., Chen, N.-K., McClernon, F. J., et al. (2012). Meditation-state functional connectivity (msFC): strengthening of the dorsal attention network and beyond. Evid. Based Complement. Alternat. Med. 2012:680407. doi: 10.1155/2012/680407

Garrison, K. A., Scheinost, D., Worhunsky, P. D., Elwafi, H. M., Thornhill, T. A., Thompson, E., et al. (2013). Real-time fMRI links subjective experience with brain activity during focused attention. Neuroimage $81,110-118$. doi: 10.1016/j. neuroimage.2013.05.030

Hasenkamp, W., and Barsalou, L. W. (2012). Effects of meditation experience on functional connectivity of distributed brain networks. Front. Hum. Neurosci. 6:38. doi: 10.3389/fnhum.2012.00038

Hasenkamp, W., Wilson-Mendenhall, C. D., Duncan, E., and Barsalou, L. W. (2012). Mind wandering and attention during focused meditation: a finegrained temporal analysis of fluctuating cognitive states. Neuroimage 59, 750-760. doi: 10.1016/j.neuroimage.2011.07.008

Himberg, J., Hyvärinen, A., and Esposito, F. (2004). Validating the independent components of neuroimaging time series via clustering and visualization. Neuroimage 22, 1214-1222. doi: 10.1016/j.neuroimage.2004.03.027

Hoaglin, D., and Iglewicz, B. (1987). Fine-tuning some resistant rules for outlier labeling. J. Am. Stat. Assoc. 82, 1147-1149. doi: 10.1080/01621459.1987. 10478551

Hofmann, S. G., Sawyer, A. T., Witt, A. A., and Oh, D. (2010). The effect of mindfulness-based therapy on anxiety and depression: a meta-analytic review. J. Consult. Clin. Psychol. 78, 169-183. doi: 10.1037/a0018555

Hölzel, B. K. (2012). "Achtsamkeitsmeditation,” in Die Große Achtsamkeits-Box, (Berlin: 5W Verlag). Available online at: http://www.5w-verlag.de/shopfitness-dvds-buecher/Gesundheit-Wellness/Die-grosse-Achtsamkeits-Box::18. html

Hölzel, B. K., Ott, U., Hempel, H., Hackl, A., Wolf, K., Stark, R., et al. (2007). Differential engagement of anterior cingulate and adjacent medial frontal cortex in adept meditators and non-meditators. Neurosci. Lett. 421, 16-21. doi: 10.1016/j.neulet.2007.04.074

Jang, J. H., Jung, W. H., Kang, D.-H., Byun, M. S., Kwon, S. J., Choi, C.-H., et al. (2011). Increased default mode network connectivity associated with meditation. Neurosci. Lett. 487, 358-362. doi: 10.1016/j.neulet.2010.10.056

Kilpatrick, L. A., Suyenobu, B. Y., Smith, S. R., Bueller, J. A., Goodman, T., Creswell, J. D., et al. (2011). Impact of mindfulness-based stress reduction training on intrinsic brain connectivity. Neuroimage 56, 290-298. doi: 10. 1016/j.neuroimage.2011.02.034

Kiviniemi, V., Starck, T., Remes, J., Long, X., Nikkinen, J., Haapea, M., et al. (2009). Functional segmentation of the brain cortex using high model order group PICA. Hum. Brain Mapp. 30, 3865-3886. doi: 10.1002/hbm.20813

Kober, H., Barrett, L. F., Joseph, J., Bliss-Moreau, E., Lindquist, K., and Wager, T. D. (2008). Functional grouping and cortical-subcortical interactions in emotion: a meta-analysis of neuroimaging studies. Neuroimage 42, 998-1031. doi: 10.1016/j.neuroimage.2008.03.059

Lewis, C., Baldassarrea, A., Committeria, G., Romania, G. L., and Corbetta, M. (2009). Learning sculpts the spontaneous activity of the resting human brain. Proc. Natl. Acad. Sci. U S A 106, 17558-17563. doi: 10.1073/pnas.0902455106

Mason, M. F., Norton, M. I., Van Horn, J. D., Wegner, D. M., Grafton, S. T., and Macrae, C. N. (2007). Wandering minds: the default network and stimulus-independent thought. Science 315, 393-395. doi: 10.1126/science. 1131295

Menon, V. (2011). Large-scale brain networks and psychopathology: a unifying triple network model. Trends Cogn. Sci. 15, 483-506. doi: 10.1016/j.tics.2011. 08.003

Menon, V., and Uddin, L. L. Q. (2010). Saliency, switching, attention and control: a network model of insula function. Brain Struct. Funct. 214, 655-667. doi: 10. 1007/s00429-010-0262-0

Riedl, V., Valet, M., Wöller, A., Sorg, C., Vogel, D., Sprenger, T., et al. (2011). Repeated pain induces adaptations of intrinsic brain activity to reflect past and 
predict future pain. Neuroimage 57, 206-213. doi: 10.1016/j.neuroimage.2011. 04.011

Seeley, W. W., Menon, V., Schatzberg, A. F., Keller, J., Glover, G. H., Kenna, H., et al. (2007). Dissociable intrinsic connectivity networks for salience processing and executive control. J. Neurosci. 27, 2349-2356. doi: 10.1523/jneurosci.558706.2007

Shaurya Prakash, R., De Leon, A. A., Klatt, M., Malarkey, W., and Patterson, B. (2013). Mindfulness disposition and default-mode network connectivity in older adults. Soc. Cogn. Affect. Neurosci. 8, 112-117. doi: 10.1093/scan/nss 115

Smith, S. M., Fox, P. T., Miller, K. L., Glahn, D. C., Fox, P. M., Mackay, C. E., et al. (2009). Correspondence of the brain's functional architecture during activation and rest. Proc. Natl. Acad. Sci. U S A 106, 13040-13045. doi: 10.1073/pnas. 0905267106

Sridharan, D., Levitin, D. J., and Menon, V. (2008). A critical role for the right fronto-insular cortex in switching between central-executive and default-mode networks. Proc. Natl. Acad. Sci. U S A 105, 12569-12574. doi: 10.1073/pnas. 0800005105

Tambini, A., Ketz, N., and Davachi, L. (2010). Enhanced brain correlations during rest are related to memory for recent experiences. Neuron 65, 280-290. doi: 10. 1016/j.neuron.2010.01.001

Taylor, V. A., Daneault, V., Grant, J., Scavone, G., Breton, E., Roffe-Vidal, S., et al. (2013). Impact of meditation training on the default mode network during a restful state. Soc. Cogn. Affect. Neurosci. 8, 4-14. doi: 10.1093/scan/nsr087
Vogt, B. A., and Laureys, S. (2005). Posterior cingulate, precuneal and retrosplenial cortices: cytology and components of the neural network correlates of consciousness. Prog. Brain Res. 150, 205-217. doi: 10.1016/s00796123(05)50015-3

Walach, H., Buchheld, N., Buttenmüller, V., Kleinknecht, N., and Schmidt, S. (2006). Measuring mindfulness-the Freiburg Mindfulness Inventory (FMI). Pers. Individ. Dif. 40, 1543-1555. doi: 10.1016/j.paid.2005.11.025

Whitfield-Gabrieli, S., Moran, J. M., Nieto-Castañón, A., Triantafyllou, C., Saxe, R., and Gabrieli, J. D. E. (2011). Associations and dissociations between default and self-reference networks in the human brain. Neuroimage 55, 225-232. doi: 10.1016/j.neuroimage.2010.11.048

Conflict of Interest Statement: The authors declare that the research was conducted in the absence of any commercial or financial relationships that could be construed as a potential conflict of interest.

Copyright (c) 2015 Doll, Hölzel, Boucard, Wohlschläger and Sorg. This is an openaccess article distributed under the terms of the Creative Commons Attribution License (CC BY). The use, distribution and reproduction in other forums is permitted, provided the original author(s) or licensor are credited and that the original publication in this journal is cited, in accordance with accepted academic practice. No use, distribution or reproduction is permitted which does not comply with these terms. 